Was ist neu in der medikamentösen Therapie? Wir halten Sie auf dem Laufenden mit Berichten von Kongressen und Symposien der pharmazeutischen Industrie.

\title{
Gastrointestinale Nebenwirkungen \\ Coxib sicherer als NSAR plus Säurehemmer
}

- 50-60\% der gastrointestinalen Blutungen unter NSAR-Langzeittherapie treten im Dünn- oder Dickdarm auf - und bleiben meist unbemerkt. Oft finden solche Blutungen bestenfalls dann Beachtung, wenn sich bereits eine Anämie entwickelt hat. Die Zugabe eines Protonenpumpenhemmers (PPI) hilft da wenig. Der bewährte Magenschutz ist unterhalb des Treitzschen Bandes wirkungslos, warnte Prof. Rainer Wigand, Frankfurt/Main.

\section{Häufigste Komplikation: Anämie}

„Die mit Abstand häufigste und klinisch relevanteste Komplikation unter einer Therapie mit traditionellen NSAR ist die Anämie“, so Wigand. Fällt der Hb unter $13 \mathrm{~g} / \mathrm{dl}$ bei Männern bzw. $12 \mathrm{~g} / \mathrm{dl}$ bei Frauen, können unerklärliche Müdigkeit, Leistungseinschränkung, Kurzatmigkeit, Zunahme der Herzfrequenz, Schwindel, Kälteüberempfindlichkeit und Brüchigkeit von Haaren und Nägeln die Patienten belasten.

Deutlich reduzieren lässt sich das Risiko durch Einsatz eines selektiven COX-2Hemmers, wie die jüngst im Lancet veröffentlichte CONDOR-Studie beweist. Laut Studienleiter Prof. Herbert Kellner, München, handelte es sich um die erste große kontrollierte Studie, die mittels neuen standardisierten Endpunkts das gastrointestinale Gesamtrisiko erfasste. Blutungen oder Perforation in Dünn- und Dickdarm wurden ebenso einbezogen wie das Absinken von $\mathrm{Hb}$ - oder Hk-Wert. Beteiligt waren fast 4500 Patienten mit Arthrose bzw. rheumatoider Arthritis und erhöhtem gastrointestinalem Risiko, die sechs Monate lang entweder ein tNSAR (Diclofenac SR 2 × 75 mg/d) plus PPI (Omeprazol $20 \mathrm{mg} / \mathrm{d}$ ) oder einen selektiven COX-2-Hemmer (Celecoxib 2 x 200 mg/d) einnahmen.

\section{Trotz PPI viermal mehr} gastrointestinale Ereignisse

Wie Kellner berichtete, waren - bei vergleichbarer Wirksamkeit - gastrointestinale Ereignisse unter tNSAR plus PPI viermal häufiger als unter dem Coxib. Signifikante Unterschiede ergaben sich bei mittleren und schweren gastrointestinalen Komplikationen gleichermaßen.
Auch Studienabbrüche infolge unerwünschter Arzneimittelwirkungen waren unter dem tNSAR signifikant häufiger (8,2 vs. 5,5\%). Während der Hb-Wert unter Celecoxib (Celebrex ${ }^{\circledR}$ ) lediglich bei 10,2\% der Behandelten unter 11,5 g/dl fiel, waren unter Diclofenac plus PPI 89,9\% betroffen. Weil in der Praxis viele Patienten den Säureblocker weglassen, dürfte sich nach Kellners Einschätzung die Überlegenheit des Coxibs dort noch deutlicher auswirken.

\section{- Ursula Einecke}

Quelle: Pressekonferenz zu CONDOR, Frankfurt/M., 24. Juni 2010 (Veranstalter: Pfizer)

\section{Reninhemmer mit besonders langer Halbwertszeit Blutdrucksenker verzeiht Einnahmefehler}

- Wie kaum eine andere Krankheit stellt die arterielle Hypertonie Anforderungen an die Compliance. Warum 30 Tage im Monat zuverlässig die Tabletten einnehmen, wenn man doch keinerlei Beschwerden hat? Wer hat schon täglich den Schlaganfall als Hypertoniefolge präsent?

Bezüglich der Compliance wurden in der Hochdrucktherapie bereits erhebliche Fortschritte erzielt: Die Antihypertensiva wurden besser verträglich, viele Substanzen müssen nur einmal täglich eingenommen werden. Doch was passiert eigentlich, wenn die Tabletteneinnahme immer mal wieder vergessen wird, was in der Praxis die Regel sein dürfte?

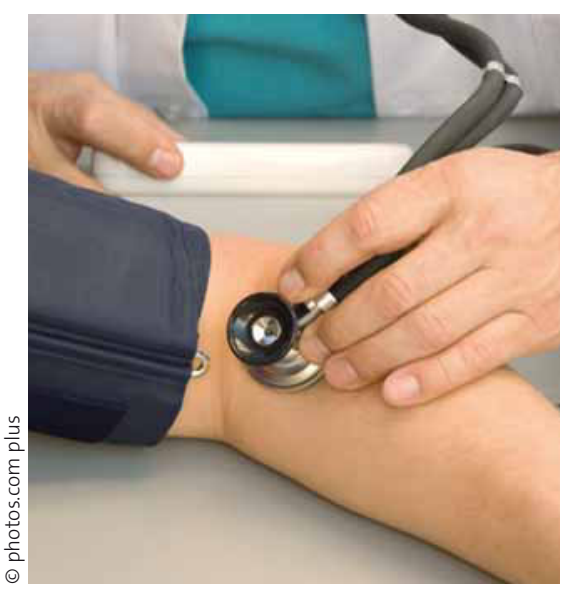

Auch wenn Aliskiren einmal vergessen wird, schießt der Blutdruck nicht in die Höhe. 
Ob am Tag der verpassten Dosis der Blutdruck wieder ansteigt, hängt von der Halbwertszeit des Blutdrucksenkers ab, sagte Prof. Rainer Düsing von der Universität Bonn. Die mit 40 Stunden besonders lange Halbwertszeit von Aliskiren hält Düsing - neben dem innovativen Wirkmechanismus und der starken Wirksamkeit - für einen Hauptvorteil des Reninantagonisten. Ob Aliskiren besser als andere Antihypertensiva Einnahmefehler verzeiht, wurde in einer „Missed Dose Study“ bei 654 Hochdruckpatienten im Vergleich zu Irbesartan und zu Ramipril getestet (Palatini et al., J Hum Hypertens 2010).

\section{„Missed dose“-Studie}

Die Patienten nahmen für je zwei Wochen zunächst die Einstiegsdosierungen und dann die vollen Dosierungen der Medikamente. In der fünften Woche wurden randomisiert Einnahmefehler simuliert - unter 24 Stunden RR-Monitoring. Ergebnis: Aliskiren schnitt am besten ab: Bei $91 \%$ der Patienten stieg der Blutdruck am Tag ohne Tabletteneinnahme nicht an. Bei Irbesartan war dies bei ca. $75 \%$ der Patienten der Fall, bei Ramipril bei $65 \%$, ein signifikanter Unterschied.

Aliskiren ist laut europäischer Leitlinie effektiv in Monotherapie und additiv wirk- sam in Kombination mit Diuretika (Rasilez $\mathrm{HCT}^{\circledR}$ ), Kalziumantagonisten, ACE-Hemmern und $\mathrm{AT}_{1}$-Blockern (Fixkombinationen mit Amlodipin und Valsartan werden 2011 erwartet). Zudem ist der Reninhemmer organprotektiv. Endpunktstudien laufen, um spezifische Vorteile der Reninhemmung zu testen. Angesichts der guten Verträglichkeit und der guten Wirksamkeit in Kombinationstherapie ist der Einsatz aber schon heute gerechtfertigt, so die europäische Hochdruckleitlinie.

- Dr. med. Dirk Einecke

Ouelle: Symposium, Weltkardiologiekongress, Peking, Juni 2010 (unterstützt von Novartis)

\section{Alzheimertherapie über die Haut „Damit kann man den Großteil
der Nebenwirkungen verhindern“}

MMW: Acetylcholinesterase-Hemmer sind die Standardtherapie bei leichter bis mittelschwerer Alzheimerdemenz. Auf dem Markt sind drei Wirkstoffe in unterschiedlichen Darreichungsformen. Wie unterscheiden sie sich?

Steinwachs: Es handelt sich um vollkommen unterschiedliche chemische Substanzen, die zwar alle Acetylcholin im Gehirn erhöhen, aber auf ganz verschiedenen Wegen. Daraus resultieren Unterschiede in der Verträglichkeit, in der zumutbaren Dosis und damit auch in der Wirkstärke.

MMW: Die Behandlung mit Cholinesterase-Hemmern wird ja relativ häufig durch gastrointestinale Nebenwirkungen eingeschränkt. Wie kann man diesem Problem am besten begegnen?

Steinwachs: Die Lösung ist die Umgehung des Gastrointestinaltrakts - durch die transdermale Applikation, wie sie mit dem Exelon ${ }^{\circledR}$-Pflaster möglich geworden ist. Gastrointestinale Probleme lassen sich zwar auch reduzieren, indem man einen oralen Cholinesterase-Hemmer langsam auftitriert. Bei der Demenztherapie hat man aber nur ein kurzes Zeitfenster, in dem man etwas erreichen kann. Wenn ich über die Titrationsphase Monate verliere, verliere ich eben auch Hirnfunktion. Mit einem Pflaster wird schneller die effiziente Dosis erreicht.

MMW: Wie erklärt sich die bessere Verträglichkeit des Pflasters?

Steinwachs: Wenn die Substanz eingenommen wird, kommt es zu Konzentrationsspitzen im Blut und im Gehirn, was zu den unangenehmen Nebenwirkungen Erbrechen, Übelkeit, Schwindel führen kann. Dagegen wird Rivastigmin bei der Resorption über die Haut ganz langsam in das System eingeflößt, sodass keine Spitzen entstehen, sondern eine komfortable Wirkdosis im mittleren Bereich. Damit kann man den Großteil der Nebenwirkungen verhindern.

MMW: Was ist bei der Anwendung des Pflasters zu beachten?

Steinwachs: Wie bei jeder Form der Demenztherapie brauchen wir natürlich einen zuverlässigen „Caregiver“. Das Pflaster wird einmal am Tag aufgeklebt. Zuvor muss das alte Pflaster entfernt werden. Um die Haut zu schonen, sollte das neue Pflaster an eine andere Stelle aufgebracht werden, und zwar an Gegenden-

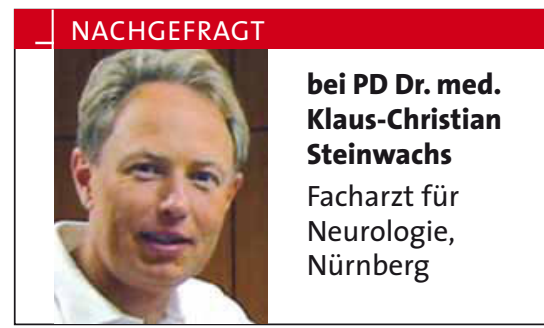

Brust, Schulter, Oberarm, Rücken -, die der Angehörige gut sehen und finden kann.

MMW: Welche Erfahrungen haben Sie bei Ihren Patienten mit dem RivastigminPflaster gemacht?

Steinwachs: Das Pflaster wird erlebt als intensive Therapie des Arztes, dazu als neuartige Therapie. Für den Patienten ist die Botschaft "der Arzt macht was". Es ist eben nicht nur noch eine Pille. Das ist entlastend, denn wir haben es bei Demenzpatienten ja mit multimorbiden Patienten zu tun. Auch der Hinweis, dass es sich um eine sanfte Therapie handelt mit wenigen Nebenwirkungen, kommt sehr gut an. Dazu gibt die Art der Anwendung dem Angehörigen die Sicherheit, dass das Medikament nicht vergessen wird. Man kann z. B. noch das Datum auf das Pflaster schreiben. Die hohe Compliance führt dazu, dass die Erfolge auch klinisch schneller zu sehen sind. Das heißt in der Regel Gleichbleiben bis leichte Verbesserung der Kognition und Alltagskompetenz im ersten halben Jahr bis Jahr.

- Interview: Dr. Beate Schumacher 